\title{
High- $E_{T}$ di-jet and three- and four-jet events in photoproduction at HERA
}

\author{
Tim Namsoo (on behalf of the ZEUS collaboration) * \\ DESY, Hamburg, Germany
}

\begin{abstract}
Two analyses of photoproduction events with hadronic jets in the final state are presented here, both of which were published by the ZEUS collaboration. The first of these studied high- $E_{T}$ di-jet final states, the second, final-states containing three- and four-jets. The di-jet analysis produced a data set to help further constrain the proton and photon PDFs. The three- and four-jet analysis produced a data set with which to test higher order perturbative QCD calculations and investigated the contribution to the cross section from multi-parton interactions.
\end{abstract}

\section{Introduction}

The study of events containing hadronic jets is useful for a multitude of reasons. Two jet based analyses are presented here [1], both published by the ZEUS collaboration using data from the HERA electron ${ }^{\mathrm{a}}$-proton $(e p)$ collider. Both analyses look at photoproduction collisions, in which the incoming electron emits a quasi-real photon $(\gamma)$ that in turn collides with the incoming proton. It is natural to think of such events as purely $\gamma p$ interactions.

The first of the analyses [2] looks at events containing at least two high-transverse energy $\left(E_{T}\right)$ jets. The aim of this analysis was to create a data set to help further constrain the proton and photon PDFs. In the case of the photon, such data is sparse, the most important being the measurement of the photon structure function, $F_{2}^{\gamma}$, measured from $\gamma e$ collisions. More diverse data is used to fit proton PDFs but presently all fits suffer from a poorly constrained gluon for $x_{p} \gtrsim 0.1$, where $x_{p}$ is the Björken scaling variable. The analysis described here attempts to gain sensitivity to the gluon density in this region.

The second analysis [3] described here is of events containing three- and four-jets. The purpose of this analysis was to produce a data set against which beyond leading-order (LO) perturbative QCD (pQCD) calculations could be tested. The pQCD description of the photoproduction environment is marginally different to that of ee, ep deep inelastic scattering (DIS) and $p p / p \bar{p}$ as the photon can behave as either a point-like electromagnetic object or a meson-like object if it fluctuates into a partonic system. The former of these two situations is referred to as direct photoproduction, the latter, resolved. A consequence of the resolved part is that some events can contain multi-parton interactions (MPIs), where more than one pair of partons from the incoming beams interact in a single event. The three- and four-jet data was used to investigate this phenomenon.

\section{High- $E_{T}$ di-jets in photoproduction}

Jets were found in the laboratory frame using the Kt-algorithm [4] and an event was selected if it had at least two jets (ordered in $E_{T}$ ) with $E_{T}^{\text {jet1 }}>20 \mathrm{GeV}$ and $E_{T}^{\text {jet2 }}>15 \mathrm{GeV}$ in the

\footnotetext{
* The support and encouragement of the DESY Directorate has been invaluable and we are indebted to the HERA machine group for their diligent efforts.

aThe word "electron" will be used to mean both electron and positron during these proceedings, unless otherwise stated.
} 
pseudorapidity, $\eta$, range, $-1<\eta^{\text {jet }}<3$, where at least one of the jets had $-1<\eta^{\text {jet }}<2.5$. The data set that was analysed had an integrated luminosity of $81.8 \mathrm{pb}-1$.

The data set was separated into direct- and resolved-photoproduction enriched regions. The two classes of events can be partially separated on the basis of the variable $x_{\gamma}^{\text {obs }}$, which approximates $x_{\gamma}$, the fraction of incoming photon momentum trans$x_{\gamma}=1$, whereas resolved events have $x_{\gamma}<1$. For this analysis, the direct-enriched region was selected to have $x_{\gamma}^{\text {obs }}>0.75$ and the remaining events were classified as resolved-like. An important distinction between the two regions is that the direct-enriched region is relatively insensitive to the photon PDF. The cross section of the resolved-enriched sample was then measured differentially and compared to a next-to-LO (NLO) pQCD prediction [5] using the CTEQ5M1 proton PDF set and each of five photon PDF sets, CJK, AFG04, SAL, GRV-HO, AFG, in order to study their relative performance.

\section{ZEUS}
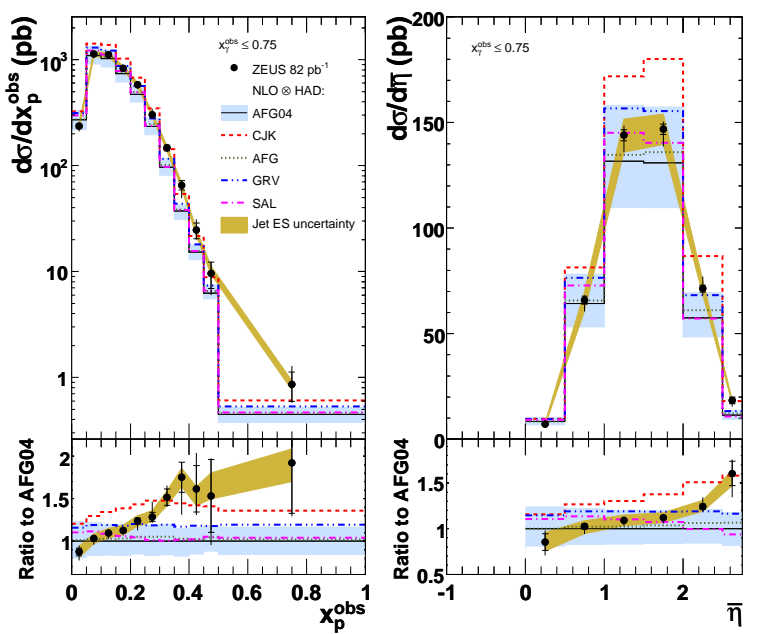

Figure 2: Di-jet cross section as a function of (a) $x_{p}^{\text {obs }}$ and (b) $\bar{\eta}$. ferred to the final state. At LO, direct events have

\section{ZEUS}

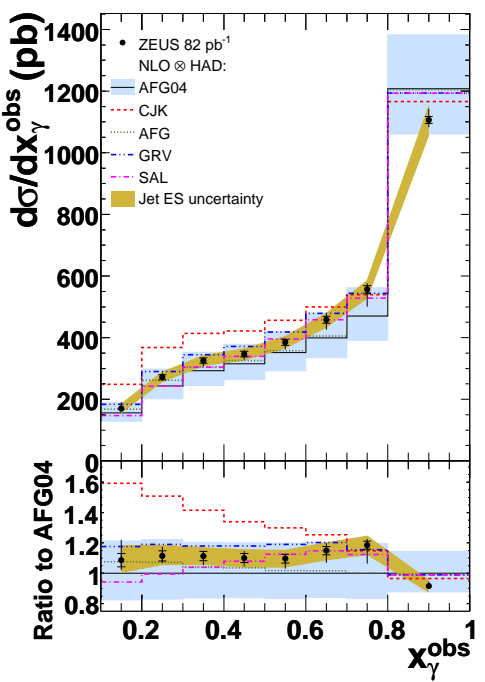

Figure 1: Di-jet cross section as a function of $x_{\gamma}^{\text {obs }}$.

Figure 1, shows the cross section of the combined directand resolved-enriched sample as a function of $x_{\gamma}^{\text {obs }}$. As expected, there is little difference between each of the predictions at high$x_{\gamma}^{\text {obs }}$ but in the low- $x_{\gamma}^{\text {obs }}$, resolvedenriched region, the differences are notable. Four of the NLO predictions lie within a $10 \%$ band around the data and, although there is some shape disagreement, largely describe the data within the uncertainties. The prediction using CJK, however, is significantly worse, overestimating the data by up to $50 \%$ at low- $x_{\gamma}^{\text {obs }}$. A notable difference is that CJK incorporates a particularly strongly rising gluon than the other PDFs.

Figure 2 shows the cross section of the resolved-enriched region as a function of $x_{p}^{\text {obs }}$, an observable that approximates $x_{p}$, and $\bar{\eta}=1 / 2\left(\eta^{j e t 1}+\eta^{j e t 2}\right)$, again compared to the same five NLO predictions. In the case of the $x_{p}^{\text {obs }}$ cross section, none of the predictions describe the data. All of the predictions 
fall off too quickly with increasing $x_{p}^{\text {obs }}$, although, in this instance, the shallower gradient obtained when CJK is used is closer to that of the data. The description of the $\bar{\eta}$ cross sections by the NLO is again mixed. The NLO cross section with CJK is clearly too large but describes better the position of the $\bar{\eta}$ peak. When the other PDFs are used, the NLO peaks at a lower value of $\bar{\eta}$ than in the data. The NLO description of the cross section at high- $\bar{\eta}$ is certainly better when CJK is used.

The analysis also identified and studied so-called "optimised" regions, where the gluon PDF uncertainties dominated other theoretical sources in the NLO calculation. Although not shown here, it is expected that the differential cross sections measured in these "optimised" regions will further constrain the gluon PDF when included in the global fits.

\section{Three- and four-jets in photoproduction}
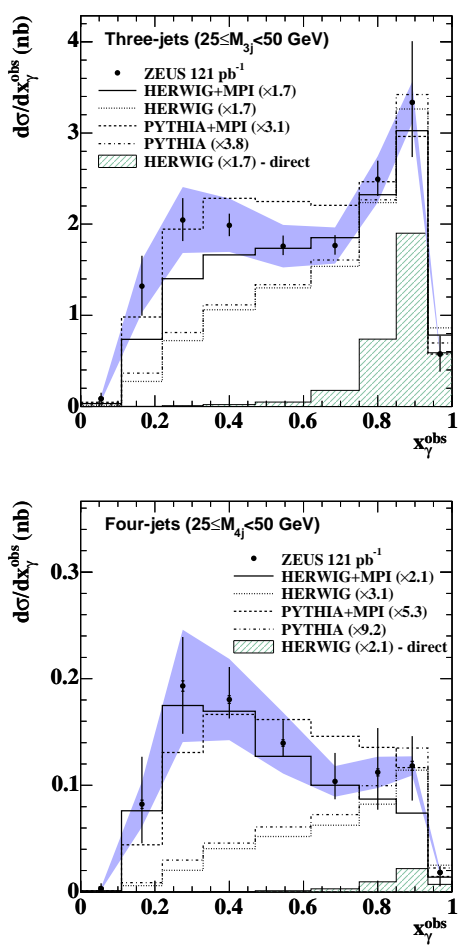

Figure 3: Three- and four-jet, lowmass cross sections as a function of $x_{\gamma}^{\text {obs }}$.

Jets were found in the laboratory frame using the Ktalgorithm and an event was selected for the threeand four-jet samples if it had at least the necessary number of jets. The jets were required to have $E_{T}^{\text {jet }}>6 \mathrm{GeV}$ and $-2.4<\eta^{\text {jet }}<2.4$. Both samples were subdivided into low- and high-mass samples containing events with $25 \leq M_{n j}<50 \mathrm{GeV}$ and $M_{n j} \geq 50 \mathrm{GeV}$, respectively, where $M_{n j}$ is the invariant mass of the $n$-jet system. The data set that was analysed had an integrated luminosity of 121 pb- 1 .

The cross section of the low- and high-mass, three- and four-jet samples were measured differentially and compared to (scaled) predictions from the Herwig [6] and Pythia [7] Monte Carlo (MC) models both with and without an MPI simulation. Figure 3 shows this comparison for the low-mass, threeand four-jet cross sections as a function of $x_{\gamma}^{\text {obs }}$. The $\mathrm{MC}$ scaling factors are given in the legend and were fixed by normalising the MC to the measured highmass cross sections. The high- $x_{\gamma}^{\text {obs }}$ region is reasonably well described by all four MC simulations, however, in the lower $x_{\gamma}^{\text {obs }}$ region, the simulations including MPIs give a far better description of the data. The MPIs are predicted to significantly augment the resolved-enriched, low- $x_{\gamma}^{\text {obs }}$ cross section and, although not shown, their influence diminishes with increasing $M_{n j}$.

The differential cross sections of the three-jet sample were also compared with a LO (for the process) pQCD calculation [8], which is shown for $\mathrm{d} \sigma / \mathrm{d} M_{3 j}$ in Fig. 4 . At the time of publication, this was the highest order pQCD calculation for photoproduction. The theory was corrected to account for hadronisation effects and the predicted influence of MPIs, and both sets of bin-by-bin correction factors can be seen in the figure. The magnitude of the MPI corrections is large (a factor 2) at low- $M_{3 j}$. The corrected theory largely describes the data within the uncertainties. Thus, 
assuming the MPI corrections are correctly modelled, the cross section in this region is relatively insensitive to beyond-LO effects but, at low- $M_{3 j}$, highly sensitive to MPIs. The MPI corrections, however, are not theoretically well constrained and so potentially they are masking the influence of higher-order processes.

\section{Summary}

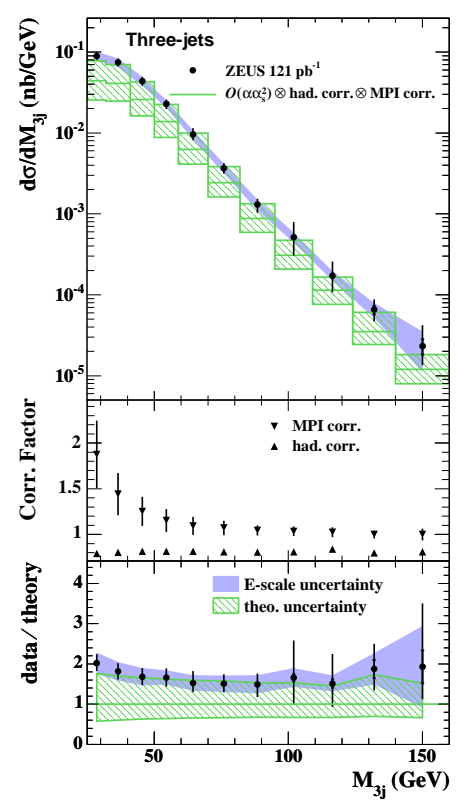

Figure 4: Three-jet low-mass cross section as a function of $M_{3 j}$.
High- $E_{T}$ di-jet cross sections have been measured in photoproduction. The cross sections are sensitive to the parameterisation of the photon PDFs. The data have been compare to an NLO calculation when 5 different photon PDFs are used. The behaviour of the NLO was similar with 4 of the PDFs but the set from CJK, which incorporates a more strongly rising gluon, was markedly different. CJK gave a poorer description of the $x_{\gamma}^{\text {obs }}$ distribution but a better description of certain features of the $x_{p}^{\text {obs }}$ and $\bar{\eta}$ distributions. None worked throughout.

Furthermore, the high- $E_{T}$ di-jet cross sections were measured in regions where the dominant uncertainty was in the gluon PDF. It is hoped such data will further constrain the gluon PDF in global fits.

The inclusive three- and four-jet cross sections have been measured in photoproduction. They have been compared to the predictions of two MC models both with and without MPIs as well as, in the case of the three-jet cross sections, a LO pQCD calculation. Both the LO pQCD and MC predictions without MPIs require augmentation at low- $M_{n j}$ and low- $x_{\gamma}^{\text {obs }}$. According to the MC models, MPIs are a possible mechanism to explain this behaviour. At high- $M_{n j}$, the influence of MPIs is predicted to be small and both LO pQCD and (scaled) MC (not shown here) describe the measured cross sections, indicating minimal NLO corrections and the validity of using PS to simulate high jet multiplicities in this region.

\section{References}

[1] Slides: http: //indico. cern. ch/contributionDisplay . py? contribId=215\&sessionId=13\&conf Id=24657

[2] S. Chekanov et al. [ZEUS Collaboration], Phys. Rev. D 76 (2007) 072011 [arXiv:0706.3809 [hep-ex]].

[3] S. Chekanov et al. [ZEUS Collaboration], Nucl. Phys. B 792 (2008) 1 [arXiv:0707.3749 [hep-ex]].

[4] S. Catani, Y. L. Dokshitzer, M. H. Seymour and B. R. Webber, Nucl. Phys. B 406 (1993) 187.

[5] S. Frixione, Z. Kunszt and A. Signer, Nucl. Phys. B 467 (1996) 399 [arXiv:hep-ph/9512328].

[6] G. Marchesini, B. R. Webber, G. Abbiendi, I. G. Knowles, M. H. Seymour and L. Stanco, Comput. Phys. Commun. 67 (1992) 465.

[7] T. Sjostrand, P. Eden, C. Friberg, L. Lonnblad, G. Miu, S. Mrenna and E. Norrbin, Comput. Phys. Commun. 135 (2001) 238 [arXiv:hep-ph/0010017].

[8] M. Klasen, T. Kleinwort and G. Kramer, Eur. Phys. J. direct C 1 (1998) 1 [arXiv:hep-ph/9712256]. 